\title{
Avaliaçấo do Brincar de Faz de Conta de Pré-Escolares: Revisão INTEGRATIVA DA LITERATURA ${ }^{1}$ \\ Evaluation of the Pretend Play by Preschool Children: an Integrative \\ REVIEW OF THE LITERATURE
}

\author{
Renata Valdívia LUCISANO² \\ Letícia De Carli NOVAES ${ }^{3}$ \\ Amanda Mota Pacciulio SPOSITO ${ }^{4}$ \\ Luzia Iara PFEIFER ${ }^{5}$
}

\begin{abstract}
RESUMO: esta revisăo integrativa da literatura objetivou identificar estudos sobre como o brincar simbólico/faz de conta de pré-escolares tem sido avaliado, quais os objetivos destes estudos e quais instrumentos têm sido utilizados para avaliar esse comportamento. Os dados foram coletados nas bases de dados CINAHL (Cumulative Index to Nursing and Allied Health Literature), PubMed, Web of Science, PsycINFO (Psychology Information), Scopus, e BVS (Biblioteca Virtual em Saúde), entre os anos 2009 e 2015, utilizando-se palavras-chave e descritores do DeCS (Descritores em Ciência da Saúde) e do MeSH (Medical Subject Headings), em diferentes combinaçóes: criança pré-escolar/child preschool; brinquedo terapêutico/play therapy; faz de conta/make believe/pretend play; jogo simbólico/symbolic play. A amostra final constituiu em 16 artigos. As publicaçóes sobre o brincar simbólico/faz de conta de pré-escolares têm sido tênues, demonstrando que ainda há muito a avançar no assunto. Dentre os instrumentos citados verifica-se que o Child Initiated Pretend Play Assessment (ChIPPA) foi o mais utilizado, estando presente em cinco artigos, envolvendo população diversa (deficiência cognitiva, lesão cerebral adquirida, paralisia cerebral e autismo). Os estudos focalizam, em sua maioria, crianças de desenvolvimento típico, objetivando obter um parâmetro para identificaçáo do desempenho do brincar simbólico/faz de conta esperado, além de demonstrar que ao se avaliar este brincar é possível verificar outros aspectos importantes para o desenvolvimento infantil, como os emocionais e cognitivos, as habilidades sociais e de comunicação, possibilitando identificar possíveis déficits destas crianças e auxiliar os profissionais em suas práticas clínicas.
\end{abstract}

PALAVRAS-CHAVE: Educação Especial. Criança Pré-Escolar. Faz de Conta. Instrumentos de Avaliação.

\begin{abstract}
: this integrative review of the literature aimed to identify studies about how the symbolic play/pretend play by preschool children has been evaluated, what the objectives of these studies are and which instruments have been used to evaluate this behavior. The data were collected in the CINAHL (Cumulative Index to Nursing and Allied Health Literature), PubMed, Web of Science, PsycINFO (Psychology Information), Scopus, and VHL (Virtual Health Library) databases between 2009 and 2015, using keywords and descriptors of DeCS (Health Science Descriptors) and MeSH (Medical Subject Headings), in different combinations: criança pré-escolar/preschool child; brinquedo terapêutico/play therapy; faz de conta/make believe/pretend play; jogo simbólico/symbolic play. The final sample consisted of 16 papers. Publications about the symbolic play/pretend play by preschoolers have been tenuous, demonstrating that there is still much to be done. Among the mentioned instruments, the Child Initiated Pretend Play Assessment (ChIPPA) was the most used, being present in five papers, involving diverse population (cognitive disability, acquired cerebral lesion, cerebral palsy and autism). Most studies focus on children of typical development, aiming to obtain a parameter to identify the performance of symbolic play / pretend play, as well as to demonstrate that when evaluating this play, it is possible to verify other important aspects for children's development, such as emotional and cognitive, social and communication skills, making it possible to identify possible deficits of these children and to assist professionals in their clinical practices.
\end{abstract}

KEYWORDS: Special Education. Preschool Children. Pretend Play. Evaluation Instruments.

\footnotetext{
${ }^{1}$ http://dx.doi.org/10.1590/S1413-65382317000200011

${ }^{2}$ Doutoranda em Neurociências pela Faculdade de Medicina de Ribeirão Preto da Universidade de São Paulo (FMRP-USP). Ribeirão Preto, SP, Brasil. renata.lucisano@usp.br

${ }^{3}$ Mestranda em Ciências pela Escola de Enfermagem de Ribeirão Preto da Universidade de São Paulo (EERP-USP). Ribeirão Preto, SP, Brasil. leticia_novaes029@hotmail.com

${ }^{4}$ Doutoranda em Ciências pela Escola de Enfermagem de Ribeirão Preto da Universidade de São Paulo (EERP-USP). Ribeirão Preto, SP, Brasil. amandamps.to@gmail.com

${ }^{5}$ Professora associada do departamento de Neurociências e Ciências do Comportamento da Faculdade de Medicina de Ribeirão Preto - FMRP-USP. Ribeirão Preto, SP, Brasil. luziara@fmrp.usp.br
} 


\section{INTRODUÇÃo}

O brincar envolve a motivação intrínseca da criança na busca do prazer através da ação, priorizando os meios (processo) em detrimento do fim (desfecho) da atividade lúdica (REZENDE, 2005). Através das brincadeiras a criança pode comunicar medo e ansiedade, adquirir um senso de controle da situação (GARIÉPY; HOWE, 2003) construindo um espaço de experimentação, de transição entre o mundo interno e externo (ALVES; BIANCHIN, 2010). Durante o brincar a criança testa e desenvolve suas habilidades, criatividade, iniciativa e autoconfiança (PEDROSA et al., 2007), já que se encontra em estado de busca, de descoberta, indagação, escolha, criação e recriação (PEREIRA, 2005), contribuindo assim para a aprendizagem e o desenvolvimento infantil, uma vez que permite a experimentação de açóes sensório-motoras, manipulação de objetos, interaçóes sociais, e a construção de representaçóes mentais (KISHIMOTO, 2008).

Na idade pré-escolar, o brincar passa a incorporar o faz de conta (também denominado de brincar simbólico por alguns autores) (STAGNITTI, 2016), o que facilita a compreensão da cultura, flexibilidade de pensamento, adaptação, aprendizado, resolução de problemas, integração de informaçóes do ambiente, desenvolvimento social, cognitivo, emocional, de habilidades físicas e linguagem (BRACIALLI; MANZINI; REGANHAN, 2004; KNOX, 2002).

Ao brincar de faz de conta a criança desempenha papéis, presentes em sua vida diária, e observa as consequências de agir de uma maneira ou de outra; ela pode internalizar regras de conduta e desenvolver um sistema de valores que orientará seu comportamento (GUIMARÁES; PEREIRA; EMMEL, 2002). O faz de conta é uma habilidade cognitiva que pode ser identificada por três importantes açóes durante o brincar: a utilização de um objeto no lugar de outro, a utilização e atribuição de alguma propriedade, ou a referência a algum objeto ou ação que está ausente (STAGNITTI, 2016).

O brincar de faz de conta está associado ao desenvolvimento das habilidades de linguagem, de negociação com pares, compreensão de conceitos, uso de símbolos no brincar e organização do tempo na brincadeira (STAGNITTI, 2009). Além de promover o desenvolvimento destas habilidades cognitivas, contribui também para a competência de auto regulação e capacidade de tomar iniciativa (NICOLOPOULOU et al., 2010).

Em sua fase mais avançada de desenvolvimento, o brincar de faz de conta, propicia que um objeto possa ser referido como se ele existisse, embora não exista (por exemplo, ingestão de alimentos invisíveis). Isso inclui habilidades para usar objetos e açóes como representações simbólicas de forma independente do contexto e aparência (descontextualização); envolver outras pessoas (incluindo figuras inanimadas) no faz de conta, e assumir papéis (decentração); combinar açóes de faz de conta em sequências cada vez mais lógicas (sequenciação); e se engajar em planejamento prévio do brincar de faz de conta (STAGNITTI, 2016).

Diante de sua importância para o desenvolvimento infantil, alteraçóes no brincar de faz de conta podem levar a problemas de aprendizagem, restriçóes na participação social e na interação com pares (STAGNITTI; UNSWORTH; RODGER, 2000) e a análise de como a criança brinca pode fornecer informações valiosas em relação às suas competências cognitivas, motoras e sociais (SANT'ANNA; BLACOVI-ASSIS; MAGALHĀES, 2008). Os instrumentos padronizados para uso junto à população infantil, incluindo aqueles que avaliam o brincar, são 
considerados de extrema importância para profissionais que trabalham com crianças e podem ser utilizados para determinar a elegibilidade das mesmas para o serviço terapêutico, monitorar progressos obtidos ao longo do processo de tratamento e auxiliar nas decisóes acerca da intervenção mais apropriada e efetiva para o caso, além de possibilitarem uma linguagem comum entre os profissionais e facilitarem a comunicação destes com a família (RICHARDSON, 2001).

Tendo em vista a importância do brincar de faz de conta para o desenvolvimento de crianças pré-escolares, este estudo teve por objetivo identificar, por meio de revisão integrativa da literatura, como esse brincar tem sido avaliado, quais os objetivos destas pesquisas e quais instrumentos têm sido utilizados para avaliar esse comportamento, buscando oferecer recursos para que os profissionais da área da saúde e educação possam incrementar ou aprimorar suas práticas com esta população.

\section{MÉTodo}

Trata-se de uma revisão integrativa da literatura, a qual tem por finalidade reunir e sintetizar resultados de pesquisas acerca de um determinado tema, de maneira sistemática, contribuindo para o aprofundamento do conhecimento da área e permitindo conclusóes acerca de uma questão específica (MENDES; SILVEIRA; GALVÃO, 2008). No geral, para a construção da revisão integrativa é preciso percorrer seis etapas distintas, similares aos estágios de desenvolvimento de pesquisa convencional (MENDES; SILVEIRA; GALVÃO, 2008). As etapas são baseadas em referenciais de estudiosos desse método, sendo o estabelecimento da hipótese ou questão da pesquisa, amostragem ou busca na literatura, categorização dos estudos, síntese do conhecimento ou apresentação da revisão, interpretação dos resultados e avaliação dos estudos incluídos na revisão (WHITTEMORE; KNAFL, 2005; BEYEA; NICOLL, 1998; BROOME, 2000; GANONG, 1987; COOPER, 1984).

Partiu-se da seguinte questão norteadora: como o brincar simbólico/faz de conta de crianças pré-escolares, com ou sem patologias tem sido focalizado nas pesquisas e intervençóes clínicas?

A coleta de dados foi realizada utilizando-se palavras-chave e descritores do DeCS (Descritores em Ciência da Saúde) e do MeSH (Medical Subject Headings), em diferentes combinaçôes: criança pré-escolar/child preschool; brinquedo terapêutico/play therapy; faz de conta/make believe/pretend play; jogo simbólico/symbolic play. Estes termos foram pesquisados nas bases de dados CINAHL (Cumulative Index to Nursing and Allied Health Literature), PubMed, Web of Science, PsycINFO (Psychology Information) e Scopus, assim como na BVS (Biblioteca Virtual em Saúde).

Para ser incluído nesta revisão, o artigo científico deveria ser obtido na íntegra; publicado entre janeiro de 2009 e dezembro de 2015; apresentar como participantes crianças pré-escolares, com desenvolvimento típico ou atípico; relatar o uso de instrumentos de avaliação do brincar simbólico/faz de conta; e ser redigido em português, inglês ou espanhol. Foram excluídos estudos de revisão ou apenas teóricos.

Após a busca, realizada por uma pesquisadora, duas outras pesquisadoras trabalharam independentemente na seleção dos artigos para inclusão, a partir inicialmente da leitura 
dos títulos e resumos dos mesmos, para se excluir as repetiçôes de resultados entre as bases e de dados. Após essa primeira seleção, uma segunda foi realizada a partir da leitura dos artigos, confirmando a manutenção ou excluindo-os. Dúvidas e discrepâncias na seleção de cada pesquisadora foram discutidas nessa segunda etapa e, posteriormente, juntamente com as demais pesquisadoras do grupo, chegando-se a uma decisão final consensual, considerando todos os critérios elencados.

Nesta análise, extraiu-se dados relativos aos autores e sua formação profissional, periódico de publicação, país de origem do estudo, tipo e objetivo da pesquisa, população participante e instrumentos de avaliação do brincar simbólico/faz de conta ${ }^{6}$ utilizados. Em seguida aprofundou-se a análise em relação ao uso dos instrumentos de avaliação, seus aspectos positivos e limitaçóes encontradas pelos autores e sua aplicabilidade em diferentes tipos de amostra. $\mathrm{Na}$ interpretação dos dados, as informações extraídas foram comparadas e os conteúdos similares foram agrupados em categorias, as quais representam as temáticas mais significativas da análise, e que serão detalhadas e discutidas na apresentação dos resultados.

\section{Resultados e discussão}

A busca eletrônica resultou em 388 estudos os quais foram revisados por duas pesquisadoras, sendo que foram excluídos 24 por seus resumos estarem indisponíveis; 35 artigos por não estarem completos ou indisponíveis na íntegra; 4 estudos por se tratarem de erratas; 18 por serem artigos de revisão; 2 estudos por estarem na língua francesa, 1 na língua hebraica e 8 na língua alemã. Além disso, foram excluídos 280 estudos que não apresentavam como foco o brincar de faz de conta e/ou crianças não pré-escolares e/ou sem um instrumento de avaliação deste brincar apresentados nos resumos ou quando lidos na íntegra. Não foram encontrados estudos que pudessem ser incluídos nos critérios desse estudo, nos anos de 2014 e 2015 . Resultando em um total de 16 estudos que compuseram esta revisão, os quais são apresentados no quadro 1.

\begin{tabular}{|c|l|l|l|l|l|}
\hline Ano & Autores & Amostra & Objetivo(s) dos estudos & $\begin{array}{l}\text { Instrumentos } \\
\text { Faz de Conta }\end{array}$ & Outros Instrumentos \\
\hline 2009 & $\begin{array}{l}\text { Uren; } \\
\text { Stagnitti }\end{array}$ & $\begin{array}{l}41 \text { crian- } \\
\text { ças de 5-7 } \\
\text { anos }\end{array}$ & $\begin{array}{l}\text { Investigar a relaçáo entre o faz de } \\
\text { conta, competências sociais e o en- } \\
\text { volvimento em atividades escolares } \\
\text { e determinar se as competências } \\
\text { sociais e o nível de envolvimento das } \\
\text { crianças podem ser inferidas a partir } \\
\text { de suas pontuaçôes no ChIPPA. }\end{array}$ & $\begin{array}{l}\text { Child Initiated } \\
\text { Pretend Play } \\
\text { Assessment }\end{array}$ & $\begin{array}{l}\text { PenIPPA) } \\
\text { Scale (PIPPS); } \\
\text { Leuven Involvement } \\
\text { Scale for Young Children } \\
\text { (LIS-YC) }\end{array}$ \\
\hline 2009 & $\begin{array}{l}\text { Quintas et } \\
\text { al. (2009) }\end{array}$ & $\begin{array}{l}32 \text { crian- } \\
\text { ças de 2-6 } \\
\text { anos }\end{array}$ & $\begin{array}{l}\text { Relacionar o brincar simbólico e } \\
\text { aspectos do desenvolvimento infan- } \\
\text { til em crianças deficientes auditivas } \\
\text { com seus pares ouvintes. }\end{array}$ & $\begin{array}{l}\text { Avaliação da } \\
\text { Maturidade } \\
\text { simbólica }\end{array}$ & Teste de Denver II \\
\hline 2009 & $\begin{array}{l}\text { McAloney; } \\
\text { Stagnitti }\end{array}$ & $\begin{array}{l}53 \text { crian- } \\
\text { ças de 4-5 } \\
\text { anos }\end{array}$ & $\begin{array}{l}\text { Investigar como o desempenho } \\
\text { de uma criança em uma avaliaçăo } \\
\text { de brincar foi relacionada com o } \\
\text { brincar social dos pares. }\end{array}$ & ChIPPA & PIPPS \\
\hline
\end{tabular}

\footnotetext{
${ }^{6}$ A partir de entấo será utilizada apenas a terminologia faz de conta para se referir a este brincar
} 


\begin{tabular}{|c|c|c|c|c|c|}
\hline Ano & Autores & Amostra & Objetivo(s) dos estudos & $\begin{array}{l}\text { Instrumentos } \\
\text { Faz de Conta }\end{array}$ & Outros Instrumentos \\
\hline 2010 & $\begin{array}{l}\text { Campos } \\
\text { et al. }\end{array}$ & $\begin{array}{l}30 \text { crian- } \\
\text { ças de 3-6 } \\
\text { anos }\end{array}$ & $\begin{array}{l}\text { Avaliar o comportamento da criança } \\
\text { antes e depois da sessão de brinque- } \\
\text { do terapêutico. }\end{array}$ & $\begin{array}{l}\text { Brinquedo Tera- } \\
\text { pêutico (BT) }\end{array}$ & Escala Comportamental \\
\hline 2010 & $\begin{array}{l}\text { Frahsek } \\
\text { et al. }\end{array}$ & $\begin{array}{l}30 \text { crian- } \\
\text { ças de } 2-2 \\
\text { anos e } 6 \\
\text { meses }\end{array}$ & $\begin{array}{l}\text { Sugerir um método que permita } \\
\text { apreciar as inter-relações entre dife- } \\
\text { rentes tipos de faz de conta. }\end{array}$ & $\begin{array}{l}\text { Pretend play } \\
\text { sequence; Ques- } \\
\text { tions on pretend } \\
\text { play }\end{array}$ & $\begin{array}{l}\text { Developmental Test (ET } \\
6-6)\end{array}$ \\
\hline 2010 & $\begin{array}{l}\text { Daunhauer } \\
\text { et al. }\end{array}$ & $\begin{array}{l}26 \text { crian- } \\
\text { ças de } 10 \text { - } \\
38 \text { meses }\end{array}$ & $\begin{array}{l}\text { Descrever o comportamento lúdico de } \\
\text { crianças institucionalizadas e explorar } \\
\text { a relaçáo entre comportamentos de } \\
\text { brincar e estado cognitivo geral. }\end{array}$ & $\begin{array}{l}\text { Two toy sets to } \\
\text { assess play }\end{array}$ & $\begin{array}{l}\text { Bayley Scales of Infant } \\
\text { Development II (BSID-II) }\end{array}$ \\
\hline 2011 & $\begin{array}{l}\text { Giacomello; } \\
\text { Melo }\end{array}$ & $\begin{array}{l}3 \text { crianças } \\
\text { de } 5-6 \\
\text { anos }\end{array}$ & $\begin{array}{l}\text { Compreender a criança instituciona- } \\
\text { lizada vítima de violência por meio } \\
\text { de sessóes de brinquedo terapêutico. }\end{array}$ & BT & $\begin{array}{l}\text { Registro de Comporta- } \\
\text { mentos }\end{array}$ \\
\hline 2011 & Pfeifer et al. & $\begin{array}{l}20 \text { crian- } \\
\text { ças de 3-6 } \\
\text { anos }\end{array}$ & $\begin{array}{l}\text { Avaliar a brincadeira autoiniciada de } \\
\text { crianças com paralisia cerebral. }\end{array}$ & ChIPPA & \\
\hline 2011 & $\begin{array}{l}\text { O'Connor; } \\
\text { Stagnitti }\end{array}$ & $\begin{array}{l}35 \text { crian- } \\
\text { ças de 5-7 } \\
\text { anos }\end{array}$ & $\begin{array}{l}\text { Investigar o brincar, comporta- } \\
\text { mento, linguagem e habilidades } \\
\text { sociais de crianças participando de } \\
\text { uma intervenção de brincar (com } \\
\text { base no programa "Learn to Play") } \\
\text { em comparação com um grupo de } \\
\text { crianças participando de atividades } \\
\text { tradicionais de sala de aula. }\end{array}$ & ChIPPA & $\begin{array}{l}\text { Goal Attainment Scaling } \\
\text { (GAS) } \\
\text { Preschool Language Scale } \\
\text { (PLS-4) } \\
\text { PIPPS }\end{array}$ \\
\hline 2012 & Fink et al. & $\begin{array}{l}3 \text { crianças } \\
\text { de } 3-6 \\
\text { anos }\end{array}$ & $\begin{array}{l}\text { Descrever a brincadeira autoinicia- } \\
\text { da de três crianças com uma lesão } \\
\text { cerebral adquirida }(\mathrm{ABI}) \text { e investigar } \\
\text { se as crianças com } \mathrm{ABI} \text { possuíam } \\
\text { déficits durante o faz de conta e, em } \\
\text { caso afirmativo, quais são eles. }\end{array}$ & ChIPPA & \\
\hline 2012 & Dereu et al. & $\begin{array}{l}17 \text { crian- } \\
\text { ças de } 17- \\
38 \text { meses } \\
\text { até seus } 4 \\
\text { anos }\end{array}$ & $\begin{array}{l}\text { Visualizar as trajetórias individuais } \\
\text { de desenvolvimento de habilida- } \\
\text { des comunicativas sociais iniciais, } \\
\text { linguagem e desenvolvimento geral de } \\
\text { uma amostra em situação de risco e } \\
\text { relacioná-las. Explorar a variabilidade } \\
\text { individual dentro e entre os grupos } \\
\text { (ASD versus casos de não-ASD) e } \\
\text { variabilidade intraindividual ao longo } \\
\text { do tempo. Abordar a questâo do } \\
\text { atraso em relaçáo ao desenvolvimento } \\
\text { desviante, comparando a taxa de } \\
\text { crescimento das habilidades sociais de } \\
\text { comunicação para cada indivíduo com } \\
\text { o observado em uma amostra norma- } \\
\text { tiva. Investigar se a escolha do método } \\
\text { de análise de dados importava. }\end{array}$ & $\begin{array}{l}\text { Test of Pretend } \\
\text { Play (ToPP) }\end{array}$ & $\begin{array}{l}\text { Mullen Scales of Early } \\
\text { Learning (MSEL), } \\
\text { ESCS. } \\
\text { PIPPS, }\end{array}$ \\
\hline
\end{tabular}




\begin{tabular}{|c|c|c|c|c|c|}
\hline Ano & Autores & Amostra & Objetivo(s) dos estudos & $\begin{array}{l}\text { Instrumentos } \\
\text { Faz de Conta }\end{array}$ & Outros Instrumentos \\
\hline 2012 & $\begin{array}{l}\text { Hoffmann; } \\
\text { Russ }\end{array}$ & $\begin{array}{l}61 \\
\text { crianças } \\
\text { de } 5-10 \\
\text { anos }\end{array}$ & $\begin{array}{l}\text { Explorar as relaçôes entre faz de } \\
\text { conta, a criatividade, a regulação da } \\
\text { emoçấo, e funcionamento executivo } \\
\text { em crianças típicas. }\end{array}$ & $\begin{array}{l}\text { Affect in Play } \\
\text { Scale (APS) }\end{array}$ & $\begin{array}{l}\text { Wisconsin Card Sorting } \\
\text { Task, Short Form (WCST- } \\
64 \text { ), } \\
\text { Emotion Regulation Che- } \\
\text { cklist (ERC) }\end{array}$ \\
\hline 2012 & Hsieh & $\begin{array}{l}58 \text { crian- } \\
\text { ças de } 6-8 \\
\text { anos }\end{array}$ & $\begin{array}{l}\text { Analisar as expressões afetivas e ima- } \\
\text { ginação em crianças com paralisia } \\
\text { cerebral em função da brincadeira } \\
\text { com materiais comuns versus brin- } \\
\text { cadeira adaptativa. }\end{array}$ & $\begin{array}{l}\text { Affect in Play } \\
\text { Scale-Brief Rat- } \\
\text { ing (APS-BR) }\end{array}$ & $\begin{array}{l}\text { The Fine Motor Scale of } \\
\text { the Peabody Develop- } \\
\text { mental Motor Scales - } 2 \\
\text { (PDMS-FM-2) }\end{array}$ \\
\hline 2013 & $\begin{array}{l}\text { Hobson } \\
\text { et al. }\end{array}$ & $\begin{array}{l}57 \text { crian- } \\
\text { ças de } 2-9 \\
\text { anos }\end{array}$ & $\begin{array}{l}\text { Testar e comparar o faz de conta } \\
\text { entre crianças com autismo, } \\
\text { transtorno do espectro autista e com } \\
\text { alteraçóes no desenvolvimento, mas } \\
\text { náo autistas. }\end{array}$ & ToPP & \\
\hline 2013 & $\begin{array}{l}\text { Kirkham } \\
\text { et al. }\end{array}$ & $\begin{array}{l}60 \text { crian- } \\
\text { ças de } 3-4 \\
\text { anos }\end{array}$ & $\begin{array}{l}\text { Investigar o desenvolvimento de } \\
\text { inter-relaçóes entre linguagem, sim- } \\
\text { bolismo gráfico e brincar simbólico, } \\
\text { ambos simultaneamente e longitu- } \\
\text { dinalmente a partir do quarto para o } \\
\text { quinto ano. }\end{array}$ & $\begin{array}{l}\text { ToPP, } \\
\text { Symbolic play }\end{array}$ & $\begin{array}{l}\text { Preschool Language Scale } \\
\text { (PSL-3), } \\
\text { Wechsler Preschool and } \\
\text { Primary Scale of Intelli- } \\
\text { gence (WPPSI-III) }\end{array}$ \\
\hline 2013 & Goods et al. & $\begin{array}{l}15 \text { crian- } \\
\text { ças de 3-5 } \\
\text { anos }\end{array}$ & $\begin{array}{l}\text { Determinar se uma intervenção } \\
\text { breve pode melhorar significativa- } \\
\text { mente a capacidade de comunicação } \\
\text { sociais das crianças em avaliaçóes } \\
\text { independentes, e quando observada } \\
\text { em sua sala de aula. }\end{array}$ & $\begin{array}{l}\text { Structured Play } \\
\text { Assessment } \\
\text { (SPA) }\end{array}$ & $\begin{array}{l}\text { Early Social Communica- } \\
\text { tion Scales (ESCS), } \\
\text { Classroom Observation } \\
\text { Measure }\end{array}$ \\
\hline
\end{tabular}

Quadro 1 - Artigos selecionados para integrar a revisão.

Fonte: elaboração própria

A maioria dos estudos foi conduzida no Brasil (QUINTAS et al., 2009; CAMPOS et al., 2010; GIACOMELLO; MELO, 2011; PFEIFER et al., 2011) e na Austrália (UREN; STAGNITTI, 2009; MCALONEY; STAGNITTI, 2009; O'CONNOR; STAGNITTI, 2011; FINK et al., 2012), com quatro estudos cada, sendo que os estudos exclusivamente Australianos apresentavam autores em comum, evidenciando terem sido elaborados pelo mesmo grupo de pesquisadores. Outros estudos foram realizados nos Estados Unidos (DAUNHAUER et al., 2010; HOFFMANN; RUSS, 2012; GOODS et al., 2013) ( $\mathrm{n}=3$ ), Inglaterra (KIRKHAM et al., 2013; HOBSON et al., 2013) $(\mathrm{n}=2)$ Alemanha (FRAHSEK et al., 2010) $(\mathrm{n}=1)$, Taiwan (HSIEH, 2012) ( $\mathrm{n}=1)$ e Bélgica (DEREU et al., 2012) ( $\mathrm{n}=1)$.

A maioria dos estudos tinha como autores terapeutas ocupacionais (UREN; STAGNITTI, 2009; MCALONEY; STAGNITTI, 2009; DAUNHAUER et al., 2010; O'CONNOR; STAGNITTI, 2011; PFEIFER et al., 2011; FINK et al., 2012) e psicólogos (HOFFMANN; RUSS, 2012; GOODS et al., 2013; KIRKHAM et al., 2013; HOBSON et al., 2013; FRAHSEK et al., 2010; DEREU et al., 2012), com seis artigos cada; os demais, foram escritos por enfermeiros (CAMPOS et al., 2010; GIACOMELLO; MELO, 2011) (dois 
artigos), fonoaudiólogos (QUINTAS et al., 2009) (um artigo), e profissional da educação não especificado (HSIEH, 2012) (um artigo).

Nestes estudos foram utilizados seis instrumentos padronizados de avaliação do brincar de faz de conta, sendo que o instrumento mais utilizado foi o Child Initiated Pretend Play Assessment (ChIPPA), estando presente em cinco estudos (UREN; STAGNITTI, 2009; MCALONEY; STAGNITTI, 2009; O'CONNOR; STAGNITTI, 2011; PFEIFER et al., 2011; FINK et al., 2012), Test of Pretend Play (TOPP), em três estudos (DEREU et al., 2012; KIRKHAM et al., 2013; HOBSON et al., 2013), Affect in Play Scale-Brief Rating (APS-Br) em dois estudos (HSIEH, 2012; HOFFMANN; RUSS, 2012), e Avaliação da Maturidade Simbólica, Pretend Play Sequence, e Structured Play Assessment (SPA) presentes em um estudo cada um (QUINTAS et al., 2009; FRAHSEK et al., 2010; GOODS et al., 2013). Além disso, alguns estudos relataram outras formas de avaliação, tais como registro de comportamentos, escalas adaptadas pelos pesquisadores, conjuntos de brinquedos, brinquedo terapêutico e observaçôes em diversos tipos de ambiente como hospitais, sala de aula, entre outros, como complemento das avaliaçóes padronizadas (CAMPOS et al., 2010; GIACOMELLO; MELO, 2011; DAUNHAUER et al., 2010).

A avaliação do brincar de faz de conta foi comparada com diversas outras avaliaçóes, as quais investigaram de forma geral o nível de desenvolvimento neuropsicomotor da criança (QUINTAS et al., 2009), ou mais especificamente a linguagem (O'CONNOR; STAGNITTI, 2011; DEREU et al., 2012; KIRKHAM et al., 2013; GOODS et al., 2013), a coordenação motora fina (PFEIFER et al., 2011; HSIEH, 2012), as competências sociais e o envolvimento em atividades escolares (UREN; STAGNITTI, 2009; MCALONEY; STAGNITTI, 2009; O'CONNOR; STAGNITTI, 2011; DEREU et al., 2012; GOODS et al., 2013), o comportamento social durante o brincar (CAMPOS et al., 2010; DAUNHAUER et al., 2010; GIACOMELLO; MELO, 2011; O'CONNOR; STAGNITTI, 2011), as inter-relaçóes entre diferentes tipos de faz de conta (FRAHSEK et al., 2010; PFEIFER et al., 2011; FINK et al., 2012; DEREU et al., 2012; HOFFMANN; RUSS, 2012; HOBSON et al., 2013; KIRKHAM et al., 2013), estado cognitivo geral (DAUNHAUER et al., 2010), a criatividade e o funcionamento executivo (HOFFMANN; RUSS, 2012), e a regulação da emoção (HOFFMANN; RUSS, 2012; HSIEH, 2012).

Os 16 estudos foram agrupados em três categorias de análise, sendo: 1) Aplicabilidade de avaliaçóes envolvendo o brincar de faz de conta em crianças com desenvolvimento típico, englobando sete estudos; 2) Aplicabilidade de avaliaçóes envolvendo o brincar de faz de conta em crianças com desenvolvimento atípico, composto por dois estudos e; 3) Aplicabilidade de avaliaçóes envolvendo o brincar de faz de conta em grupos comparativos, com sete estudos.

\subsection{APLICABILIDADE DE AVALIAÇÓES ENVOLVENDO O BRINCAR DE FAZ DE CONTA EM CRIANÇAS COM DESENVOLVIMENTO TÍPICO}

Dentre os estudos selecionados, sete analisaram o brincar de faz de conta de crianças de desenvolvimento típico: GIACOMELLO; MELO (2011); CAMPOS et al. (2010); DAUNHAUER et al. (2010); FRAHSEK et al. (2010); MCALONEY; STAGNITTI (2009); UREN; STAGNITTI (2009) e HOFFMANN; RUSS (2012). 
A faixa etária dos participantes foi de 10 meses a 10 anos e o tamanho da amostra variou entre três e 61 crianças, sendo que apenas um trabalho (GIACOMELLO; MELO, 2011) apresentou três estudos de caso enquanto os demais avaliaram 26 crianças ou mais. Quanto ao método utilizado, um estudo (GIACOMELLO; MELO, 2011) utilizou a análise qualitativa fenomenológica e os demais estudos (CAMPOS et al., 2010; DAUNHAUER et al., 2010; FRAHSEK et al., 2010; MCALONEY; STAGNITTI, 2009 e UREN; STAGNITTI, 2009; HOFFMANN; RUSS, 2012) foram de caráter descritivo, observacional e não experimental.

Referente aos instrumentos de avaliação do brincar de faz de conta desta categoria foi utilizado o CHIPPA (MCALONEY; STAGNITTI, 2009 e UREN; STAGNITTI, 2009), Pretend Play Sequence (FRAHSEK et al., 2010) e APS (HOFFMANN; RUSS, 2012).

Dois estudos envolveram crianças de desenvolvimento típico que vivem em ambientes domésticos (FRAHSEK et al., 2010; HOFFMANN; RUSS, 2012), enquanto que os demais cinco estudos analisaram o brincar de faz de conta de crianças em instituiçóes (abrigo, hospital ou escola) (GIACOMELLO; MELO, 2011; CAMPOS et al., 2010; UREN; STAGNITTI, 2009; MCALONEY; STAGNITTI, 2009; DAUNHAUER et al., 2010).

O estudo de Giacomello e Melo (2011) apresentou como resultado conteúdos narrados pelas crianças durante o brincar, que evidenciaram situaçôes de seu cotidiano, ora de modo tranquilo, ora de modo violento, abordando questóes sobre a instituição de abrigamento e a vinculação com os profissionais que trabalham no abrigo e também com os seus familiares.

No estudo de Campos et al. (2010) as crianças apresentaram mudança de comportamento após a sessão de brinquedo terapêutico, de não interesse e baixa interação da criança relacionadas a movimentar parte do corpo e expressar emoções, como seriedade, dirigir o olhar a um local rapidamente como se estivessem esperando algo; para maior interação como mudar de posição, deslocar-se com uma finalidade, observar atentamente o que viam e expressar emoçóes, como alegria.

Os resultados do estudo de Daunhauer et al. (2010) sugerem que o desenvolvimento cognitivo está fortemente relacionado com comportamentos de brincar $(\mathrm{r}=0,78, \mathrm{p} \leq .01)$. Esta descoberta também sugere que as avaliaçóes do brincar podem ser usadas por terapeutas que tentam avaliar as crianças recém-adotadas em instituiçóes que são difíceis de se envolverem em testes padronizados, mas capazes de se engajarem no brincar de forma livre.

O método proposto no estudo de Frahsek et al. (2010), ainda não cumpre todos os critérios psicométricos de um teste, oferecendo somente evidências parciais de objetividade e validade, sem analisar a confiabilidade do método.

Tanto o estudo de McAloney e Stagnitti (2009) quanto o de Uren e Stagnitti (2009), encontraram correlaçóes positivas e significativas entre os instrumentos relacionados, em que os objetivos propostos pelas avaliaçóes PIPPS e LIS-YC podem ser inferidos pelas pontuações obtidas no ChIPPA.

E, por fim, no estudo de Hoffmann e Russ (2012), foi encontrado correlação significativa entre o faz de conta, a criatividade e a regulação da emoção, e não foi encontrada relação significante com funcionamento executivo. 


\subsection{APLICABILIDADE DE AVALIAÇÓES ENVOLVENDO O BRINCAR DE FAZ DE CONTA EM CRIANÇAS COM DESENVOLVIMENTO ATÍPICO}

As duas pesquisas relacionadas a essa categoria (PFEIFER et al., 2011; FINK et al., 2012) aplicaram avaliaçóes em diferentes populaçóes-alvo, entretanto, utilizaram o mesmo instrumento de avaliação (ChIPPA) e avaliaram crianças na mesma faixa etária (três a seis anos).

O estudo de Pfeifer et al. (2011), analisou o brincar espontâneo de 20 crianças com paralisia cerebral, distribuídas nos cinco níveis de classificação do Gross Motor Function Classification System (GMFCS) (PALISANO et al., 1997). Já o estudo de Fink et al. (2012) analisou o brincar de faz de conta autoiniciado de três crianças com lesão cerebral adquirida (meningite, malformação arteriovenosa e acidente de veículo motor) de 15 dias a dois meses de lesão. Quanto ao método utilizado, ambos foram estudos aplicados e não-experimentais.

Houve correlaçóes negativas significativas entre o nível de gravidade motora das crianças e sua elaboração no brincar imaginativo-convencional e simbólico, no estudo de Pfeifer et al. (2011). Isto indica que as crianças com maiores limitações motoras têm diminuída suas habilidades para o brincar. Nessa amostra, 35\% das crianças mostraram estilos de brincar típicos, identificados por bom escore em elaborar ações na brincadeira, número de substituiçóes de objetos e capacidade de autoiniciar o brincar. Os perfis de déficits no faz de conta que podem ser esperados em crianças com paralisia cerebral foram descritos. Além disso, os autores propuseram sugestóes e indicações para intervenção terapêutica para melhorar o desempenho destas crianças na brincadeira.

Já no estudo de Fink et al. (2012), uma criança pontuou acima do intervalo esperado para crianças da sua idade, enquanto que as outras duas pontuaram abaixo do intervalo, indicando uma ampla variedade de habilidades cognitivas que envolvem planejamento sequencial, resolução de problemas, linguagem e compreensão social durante o brincar de faz de conta. Nenhuma das crianças conseguiu completar o tempo esperado da avaliação, podendo ser explicada pela fadiga cognitiva apresentada nessa condição clínica.

As autoras ainda descrevem os estilos do brincar, sendo que duas crianças apresentaram estilo de brincadeira de narrativa, ou seja, um estilo de brincar considerado típico em que a criança apresenta açóes elaboradas no brincar com uma pontuação dentro ou acima do esperado, substituiçôes de objetos e uma baixa pontuação em ações imitadas. Porém, uma das crianças apresentou um brincar baseado num roteiro de filme ao invés de autoiniciar a brincadeira, com açóes repetitivas em sua narrativa, e a outra criança autoiniciou a brincadeira contando uma história, elaborando sequências lógicas e criando cenas de forma organizada, sem se basear em uma história já conhecida. E a terceira criança apresentou um estilo de brincar considerado atípico, sendo chamado de funcional, ou seja, a criança consegue relacionar os objetos no brincar, no entanto apresenta incapacidade para estender o seu brincar e/ou em elaborar sequências narrativas e podem apresentar risco de dificuldades escolares (FINK et al., 2012). 


\subsection{APLICABILIDADE DE AVALIAÇÓES ENVOLVENDO O BRINCAR DE FAZ DE CONTA EM GRUPOS COMPARATIVOS}

Foram analisados sete estudos que utilizaram grupos comparativos para avaliar o desempenho ou desenvolvimento do brincar de faz de conta em crianças pré-escolares com e sem distúrbios no desenvolvimento.

Dentre estes estudos, três compararam o brincar de faz de conta de crianças de desenvolvimento típico e crianças com Transtorno do espectro autista (GOODS et al., 2013; DEREU et al., 2012; HOBSON et al., 2013); os outros estudos envolveram crianças com paralisia cerebral (HSIEH, 2012); déficit cognitivo (O'CONNOR; STAGNITTI, 2011); deficiência auditiva (QUINTAS et al., 2009) e crianças de desenvolvimento típico (KIRKHAM et al., 2013). A faixa etária variou entre 17 meses a nove anos de idade. A amostra variou entre 15 e 60 participantes. Quanto ao método utilizado, quatro estudos (QUINTAS et al., 2009; DEREU et al., 2012; HOBSON et al., 2013; KIRKHAM et al., 2013) foram de caráter descritivo, observacional e não experimental, composto por dois grupos e três foram estudos clínicos comparando os resultados pré e pós intervenção lúdica (HSIEH, 2012; O’CONNOR; STAGNITTI, 2011, GOODS et al., 2013).

Referente aos instrumentos de avaliação do brincar de faz de conta foram utilizados o APS-Br (HSIEH, 2012), CHIPPA (O'CONNOR; STAGNITTI, 2011), SPA (GOODS et al., 2013), ToPP (DEREU et al., 2012; HOBSON et al., 2013; KIRKHAM et al., 2013) e Avaliação da Maturidade Simbólica (QUINTAS et al., 2009).

Os resultados do estudo de Hsieh (2012), apontaram que as crianças com desenvolvimento típico apresentaram significativamente mais expressóes emocionais e imaginação do que as crianças com Paralisia Cerebral (PC), entretanto, a média das pontuaçóes do APS$\mathrm{BR}$, para o grupo de crianças com PC, aumentaram com a atividade adaptada, demonstrando mudança positiva no comportamento imaginativo, melhor manipulação, sendo observados comportamentos mais bem-humorados e simbólicos.

No estudo de Quintas et al. (2009), sugere-se, a partir dos resultados, que o grupo de pesquisa (GP) tem déficits nas habilidades cognitivas avaliadas e demonstra uma dificuldade na percepção da realidade em que estão inseridos resultando numa maior dificuldade de aprendizagem. O grupo controle (GC) apresentou melhor desempenho nas provas de imitação, sendo que nos esquemas gestuais simples, a diferença entre os desempenhos foi maior. Sugerese também que o GP tem potencial para aprender e desenvolver suas habilidades cognitivas, dependendo de fatores externos como estimulação, condiçóes sociais e ambientais. Por análise quantitativa, os grupos apresentam desempenho semelhante na Avaliação da Maturidade Simbólica, entretanto, em análise qualitativa mais detalhada é possível verificar que o GP apresentou desempenho inferior ao GC, principalmente em relação ao tipo de frequência de jogos mais complexos.

Em resumo, no estudo de Dereu et al. (2012), o resultado aponta que com relação à imitação, as crianças do grupo sem autismo apresentaram melhor performance durante o processo de avaliação, comparado às crianças com autismo, porém, crianças com autismo mostraram maior curva de mudança, resultando em performances similares para ambos grupos. Com relação ao brincar de faz de conta, as crianças com autismo apresentaram valores inferiores no 
ToPP do que crianças sem autismo, entretanto a interação do efeito tempo versus grupo não foi estatisticamente significativa. Com relação à articulação da atenção, o tempo apresentou marginalmente significância, mas não houve diferença significativa entre os grupos. Por fim, os autores pressupóem que é difícil esboçar uma visão global do desenvolvimento sócio-comunicativo de crianças com autismo, por causa das grandes diferenças inter-individuais e intra-individuais dessas habilidades. Os resultados apontaram que não é possível abordar a questão do desenvolvimento desviante versus o desenvolvimento atrasado, por meio da comparação da taxa de crescimento das habilidades comunicativas sociais, baseado em análises que comparam grupos de crianças com autismo e grupo de crianças com outras desordens do desenvolvimento.

Como resultados, o estudo de Hobson et al. (2013), encontraram que as crianças com autismo apresentaram escores significativamente mais baixos do que aquelas sem autismo, na medida composta de qualidades lúdicas aplicada ao ToPP, bem como relativa escassez de pretexto lúdico. Houve correlação entre a comunicação/habilidades de interação social e pretexto lúdico através de uma gama mais ampla de crianças com deficiências de desenvolvimento; deficiências na comunicação/interação social estão associadas com limitaçóes na qualidade de jogo, mesmo quando habilidades do jogo formal (como avaliado pelo ToPP) sáo levados em conta; deficiências na comunicação e interação social foram correlacionados com menor pontuação no ToPP. Entre os grupos, as preferências de brincadeiras estão correlacionadas as diferenças individuais na interação sócio-comunicativa, mesmo quando a pontuação do ToPP é considerada. Limitação na criatividade e imitação entre as crianças com autismo se relacionam com sua comunicação e engajamento interpessoal restrito.

Também no estudo de O'Connor e Stagnitti (2011), os resultados apontaram que ao longo dos seis meses de intervenção lúdica, as crianças apresentaram menos dificuldades, melhora no comportamento, maior sociabilidade e interatividade e menor desconectividade com os pares. Ao serem comparadas ao grupo que não sofreu intervenção, os ganhos foram significativos no brincar de faz de conta, menor produção de imitaçóes e melhora significativa na pontuação total de linguagem.

No estudo de Goods et al. (2013), os resultados apontaram melhora da diversidade de jogos em uma medida experimental e alguma indicação de diminuição do tempo de desengajamento na sala de aula. Os resultados foram mistos, mas também promissores para melhorar gestos solicitados e, apesar de almejado, não houve melhora em gestos de atenção.

E por fim, no estudo de Kirkham et al. (2013), os resultados encontrados na primeira análise sugerem que as habilidades gráficas, de linguagem e do brincar de faz de conta em crianças são inter-relacionadas e desenvolvem-se em paralelo até o momento em que os dados foram analisados, ou seja, aos quatro anos de idade. A análise longitudinal encontrou que não há relaçóes preditivas nem entre o brincar de faz de conta ou grafia simbólica no quarto ano de vida e linguagem no quinto ano de vida. Os resultados também sugerem que o domínio inicial da capacidade de processar estímulos representacionais existe até por volta dos quatro anos, depois a linguagem assume o papel de estruturação e desenvolvimento guiando outros domínios simbólicos, potencialmente através de um processo semelhante a re-descrição representacional. Além disso, nem o brincar de faz de conta, nem tarefas de simbolismo gráfico conseguiram prever os conhecimentos linguísticos das crianças ao longo do tempo, assim, parece que, durante 
o período pré-escolar, o brincar de faz de conta e simbolismo gráfico podem não oferecer uma janela através da qual a competência linguística das crianças possa ser avaliada.

\section{Conclusấo}

Esta revisão demonstra que na literatura há poucos estudos focalizando o brincar de faz de conta de crianças pré-escolares sobre essa temática. A análise da produção científica sobre os estudos acerca do brincar de faz de conta de crianças pré-escolares, objetivo desse estudo, proporcionou uma reflexão quanto à atenção que tem sido dada a este brincar como facilitador do desenvolvimento de habilidades e competências durante a primeira infância.

O brincar de faz de conta mostra-se como um mediador eficaz na aprendizagem, pois é a linguagem que a criança conhece, possibilitando a expressáo de seus sentimentos, desejos, e entendimento de suas capacidades de representar o mundo experienciado e vivido.

Os profissionais de saúde e da educação necessitam avaliar crianças pré-escolares e, para tanto, é importante que conheçam e apresentem domínio na utilização de protocolos de avaliação que visem auxiliar na compreensão do brincar destas crianças, facilitando a utilização deste como meio e fim terapêutico no enfoque das ocupações do brincar, lazer e participação social, no direcionamento de informaçóes e intervençóes necessárias ao desenvolvimento, considerando as etapas lúdicas pelas quais a criança transita.

Além disso, é necessária a realização de outros estudos que tenham como foco o brincar de crianças pré-escolares, sendo essa, uma importante área de pesquisa que embasa a prática de profissionais da saúde e da educação.

\section{REFERÊNCIAS}

ALVES, L.; BIANCHIN, M.A. O jogo como recurso de aprendizagem. Revista Psicopedagogia, São Paulo, v.27, n.83, p.282-287, 2010.

BEYEA, S.C.; NICOLL, L.H. Writing an integrative review. AORN Journal, Denver, v.67, n.4, p.87780, 1998.

BRACCIALLI, L.M.P.; MANZINI, E.J.; REGANHAN, W.G. Contribuição de um programa de jogos e brincadeiras adaptados para a estimulação de habilidades motoras em alunos com deficiência física. Temas Sobre Desenvolvimento, São Paulo, v.13, p.37-46, 2004.

BROOME, M.E. Integrative literature reviews for the development of concepts. In: RODGERS, B.L.; KNAFL, K.A. (Org.). Concept development in nursing: foundations, techniques and applications. Philadelphia: W.B Saunders Company, 2000. p.231-50.

CAMPOS, M.C.; RODRIGUES, K.C.S.; PINTO, M.C.M. Evaluation of the behavior of the preschool one just admitted in the unit of pediatrics and the use of the therapeutic toy. Einstein, v.8, n.1, p.10-7, 2010.

COOPER, H.M. The integrative research review: a systematic approach. Beverly Hills: Sage Pulications, 1984.

DAUNHAUER, L.A. et al. Play and cognition among young children reared in an institution. Physical \& Occupational Therapy in Pediatrics, v.30, n.2, 2010. 
DEREU, M. et al. Exploring individual trajectories of social communicative development in toddlers at risk for autism spectrum disorders. Research in Autism Spectrum Disorders, v.6, p.1038-1052, 2012.

FINK, N.; STAGNITTI, K.; GALVIN, J. Pretend play of children with acquired brain injury: an exploratory study. Developmental Neurorehabilitation, London, v.15, n.5, p.336-342, 2012.

FRAHSEK, S. et al. Assessing different aspects of pretend play within a play setting: Towards a standardized assessment of pretend play in young children. British Journal of Developmental Psychology, v. 28, p.331-345, 2010.

GANONG, L.H. Integrative reviews of nursing research. Research in Nursing \& Health, v.20, n.1, p.1$11,1987$.

GARIÉPY, N.; HOWE, N. The therapeutic power of play: examining the play of young children whith leukaemia. Child: Care, Health and Development, v.29, n.6, p.523-537, 2003.

GIACOMELLO, K.J.; MELO, L.L Do faz de conta à realidade: compreendendo o brincar de crianças institucionalizadas vítimas de violência por meio do brinquedo terapêutico. Ciência \& Saúde Coletiva, v.16, n.1, p.1571-1580, 2011.

GOODS, K.S. et al. Preschool based JASPER intervention in minimally verbal children with autism: Pilot RCT. J Autism Dev Disord, v. 43, p.1050-1056, 2013.

GUIMARÁES, A.E.O.; PEREIRA, E.C.; EMMEL, M.L.G. A brincadeira simbólica nas situaçóes lúdicas de crianças portadoras de necessidades especiais e crianças normais. Temas sobre Desenvolvimento, Sorocaba, v. 11, p. 5-13, 2002.

HOBSON, J.A. et al. The relation between social engagement and pretend play in autismo. British Journal of Developmental Psychology, v.31, p.114-127, 2013.

HOFFMAN, J.; RUSS, S. Pretend play, creativity, and emotion regulation in children. Psychology of Aesthetics, Creativity, and the Arts, v.6, n.2, p.175-184, 2011.

HSIEH, H.C. Effectiveness of adaptive pretend play on affective expression and imagination of children with cerebral palsy. Research in Developmental Disabilities, v.33, p.1975-1983, 2012.

KIRKHAM, J.; STEWARTB, A.; KIDD, E. Concurrent and longitudinal relationships between development in graphic, language and symbolic play domains from the fourth to the fifth year. Inf. Child. Dev., v.22, p.297-319, 2013.

KISHIMOTO, T.M. Jogo, brinquedo, brincadeira e a educação. 11.ed. São Paulo: Cortez, 2008.

KNOX, S.H. Avaliação da recreação e lazer. In: NEISTADT, M.E.; CREPEAU, E.B. (Org.). Willard \& Spackman - Terapia ocupacional. Rio de Janeiro: Guanabara Koogan, 2002. p.195-201.

McALONEY, K; STAGNITTI, K. Pretend play and social play: The concurrent validity of the ChildInitiated Pretend Play Assessment. International Journal of Play Therapy, Clovis, v.18, n.2, p.99-113, 2009.

MENDES, K.D.S.; SILVEIRA, R.C.C.P.; GALVÃO, C.M. Revisão integrativa: método de pesquisa para a incorporação de evidências na saúde e na enfermagem. Texto Contexto Enferm, Florianópolis, v.17, n.4, p.758-64, 2008.

NICOLOPOULOU, A. et al. Using the transformative power of play to educate hearts and minds: from Vygotsky to Vivian Paley and Beyond. Mind, Culture and Activity, v.17, p.42-58, 2010. 
O'CONNOR, C.; STAGNITTI, K. Play, behaviour, language and social skills: the comparison of a play and a non-play intervention within a specialist school setting. Research in Developmental Disabilities, Elmsford, v.32, n.3, p.1205-1211, 2011.

PALISANO R. et al. Development and reliability of a system to classify gross motor function in children with cerebral palsy. Dev Med Child Neurol., v.39, n.4, p.214-23, 1997.

PEDROSA, A.M. et al. Diversão em movimento: um projeto lúdico para crianças hospitalizadas no serviço de oncologia pediátrica do instituto materno infantil Prof. Fernando Figueira, IMIP. Revista Brasileira Saúde Materno Infantil, Recife, v.7, n.1, p. 99-106, 2007.

PEREIRA, E.T. Brincar e criança. In: CARVALHO, A. et al. (Org.). Brincar (es). Belo Horizonte: Editora UFMG, 2005. p.17-27.

PFEIFER, L.I. et al. Pretend play of children with cerebral palsy. Physical and Occupational Therapy in Pediatrics, New York, v.31, n.4, p.1-13, 2011.

QUINTAS, T.A. et al. Caracterização do jogo simbólico em deficientes auditivos: estudo de casos e controles. Pró-Fono Revista de Atualização Cientifica, v.21, n.4, p.303-8, 2009.

REZENDE, M. O brincar sob a perspectiva da terapia ocupacional. In: CARVALHO, A. et al. (Org.). Brincar (es). Belo Horizonte: Editora UFMG, 2005. p.17-27.

RICHARDSON, P.K. Use of standardized tests in pediatric practice. In: CASE-SMITH, J. (Org.). Occupational therapy for children. St. Louis, MI: Mosby, 2001. p.246-275.

SANT'ANNA, M.M.M.; BLACOVI-ASSIS, S.M.; MAGALHÃES, L.C. Adaptação transcultural dos protocolos de avaliação do Modelo Lúdico. Revista de Terapia Ocupacional da Universidade de São Paulo, São Paulo, v.19, p.34-47, 2008.

STAGNITTI, K. Children and pretend play. In: STAGNITTI, K.; COOPER, R. (Eds.). Play as therapy: Assessment and therapeutic interventions. London: Jessica Kingsley Publishers, 2009.

STAGNITTI, K. Play therapy for school-age children with high functioning autism. In: DREWES, A.; SCHAEFER, C. (Eds.). Play therapy in middle childhood. New York: American Psychological Association, 2016. p.237-255.

STAGNITTI, K.; UNSWORTH, C.; RODGER, S. Development of an assessment to identify play behaviours that discriminate between the play of typical preschoolers and preschoolers with preacademic problems. Canadian Journal of Occupational Therap, v.67, p.291-303, 2000.

UREN, N.; STAGNITTI, K. Pretend play, social competence and learning in preschool children. Australian Occupational Therapy Journal. v.56, p.33-40, 2009.

WHITTEMORE, R.; KNAFL, K. The integrative review: updated methodology. J Adv Nurs, v.52, n.5, p. 546-53, 2005.

Recebido em: 12/06/2016

Reformulado em: 26/05/2017

Aprovado em: 31/05/2017 